\title{
Leaf Segmentation and Tracking Using Probabilistic Parametric Active Contours
}

\author{
Jonas De Vylder ${ }^{1 *}$, Daniel Ochoa ${ }^{1,2}$, Wilfried Philips ${ }^{1}$, Laury Chaerle ${ }^{3}$, and \\ Dominique Van Der Straeten ${ }^{3}$ \\ ${ }^{1}$ Department of Telecommunications and Information Processing, \\ IBBT - Image Processing and Interpretation, Ghent University \\ ${ }^{2}$ Facultad de Ingenieria en Electricidad y Computacion, Escuela Superior Politecnica del Litoral \\ ${ }^{3}$ Department of Physiology, Laboratory of Functional Plant Biology, Ghent University \\ jonas.devylderetelin.ugent.be \\ http://telin.ugent.be/ jdvylder
}

\begin{abstract}
Active contours or snakes are widely used for segmentation and tracking. These techniques require the minimization of an energy function, which is generally a linear combination of a data fit term and a regularization term. This energy function can be adjusted to exploit the intrinsic object and image features. This can be done by changing the weighting parameters of the data fit and regularization term. There is, however, no rule to set these parameters optimally for a given application. This results in trial and error parameter estimation. In this paper, we propose a new active contour framework defined using probability theory. With this new technique there is no need for ad hoc parameter setting, since it uses probability distributions, which can be learned from a given training dataset.
\end{abstract}

\section{Introduction}

With a constantly increasing demand for food it becomes necessarily to optimize agricultural planning, e.g. to plant the best type of plants and use the best fertilizers for a given field with a specific soil, expected weather, etc. This off course assumes it is known what the best plant type is for a specific field. This has led to the development of biological laboratories which quantitatively measure the development of plants under the influence of specific stress factors, e.g. wind, lack of nutrients, etc. A common feature to evaluate the well-being of a plant is to periodically measure the average leaf temperature using a thermal camera.

The reliable measuring of average leaf temperature in thermal images is a time consuming task. It demands skilled technicians who spend time identifying and delineating objects of interest in the image. Although interactive software can ease this work, this approach becomes impractical when the measurements have to be monitored over time for a large variety of plant types. This paper proposes an automated technique to segment leaves and measure its average temperature in thermal images. An interesting approach to segment objects is based on probability theory [1,2]. In this work, a new Bayesian technique is proposed. This new technique combines the Bayesian framework 
with the popular active contour model, an extensively studied segmentation and tracking framework used in computer vision.

In the active contour framework, an initial contour is moved and deformed in order to minimize a specific energy function. This energy function should be minimal when the contour is delineating the object of interest, e.g. a leaf. Two main groups can be distinguished in the active contour framework: one group representing the active contour explicitly as a parameterized curve and a second group which represents the contour implicitly using level sets. In the first group, also called snakes, the contour generally converges towards edges in the image $[3,4,5]$. The second group generally has an energy function based on region properties, such as the intensity variance of the enclosed segment [6,7]. These level set approaches have gained a lot of interest since they have some benefits over snakes. They can for example easily change their topology, e.g. splitting a segment into multiple unconnected segments. Recently an active contour model has been proposed with a convex energy function, making it possible to define fast global optimizers $[8,9]$.

Unfortunately do the level set approaches assume certain prior knowledge about the regions defined, e.g. the variance of intensity in the segments should be minimal. These kind of assumptions are unfortunately not always valid and might bias the temperature measurements of leaves. Therefore we will base our new active contour model on the snake approach. This doesn't form a problem since leaves don't divide and therefore we don't need the variable topology of level set active contours. Although snakes don't have global optimizers, several optimizations techniques have been proposed and proven useful for segmentation and tracking. In order not to converge to local optima, one or more regularization terms are incorporated in the energy function. The influence of these regularization terms can be tuned using a set of weighting parameters. This tuning is generally done by trial and error, which is a time consuming and error prone approach. Even after manually tuning, the parameters might not be optimal since the segmentation quality in function of these weighting parameters generally is not a convex function. So only by exploring the full parameter space one can be sure to find the optimal parameters. We propose a new active contour framework based on probability theory. Instead of exhaustively searching optimal weighting parameters, the proposed method uses prior knowledge about the probability of certain features, e.g. edges. It also removes the linear influence of image features, i.e. it is not because the gradient of an edge is twice as large as another edge, that it is twice as likely to be the true border of an object. This is especially important if you are segmenting leaves in noisy images with other objects.

This paper is arranged as follows. The next section provides a detailed description of parametric active contours. Both the classical and gradient vector flow snakes are explained. In section 3 our proposed algorithm is presented. Section 4 elaborates on the results and compares the proposed methods with the classical snakes. Finally, section 5 recapitulates and lists some future research possibilities. 


\section{Active Contours}

\subsection{Snakes}

The classical snake model proposed by Kass et al. [4], defines the active contour as a parametric curve, $\mathbf{r}(s)=(x(s), y(s))$ with $s \in[0,1]$, that moves in the spatial domain until the energy functional in Eq. (1) reaches its minimum value.

$$
E[\mathbf{r}]=E_{\text {int }}(\mathbf{r}(s))+E_{\text {ext }}(\mathbf{r}(s))
$$

$E_{\text {int }}[$.$] and E_{\text {ext }}[$.$] represent the internal and external energy, respectively correspond-$ ing to a regularization and a data fit term. A common internal energy function that enforces smoothness along the contour is defined as follows:

$$
E_{\text {int }}[\mathbf{r}(s)]=\frac{1}{2} \int \alpha\left|\frac{\partial \mathbf{r}(s)}{\partial s}\right|^{2}+\beta\left|\frac{\partial^{2} \mathbf{r}(s)}{\partial d s^{2}}\right|^{2} d s
$$

where $\alpha$ and $\beta$ are weighting parameters. The first term, also known as tension energy, prevents the snake to "stretch" itself too much, thus avoid being attracted to isolated points. The second term, known as bending energy, prevents the contour of developing sharp angles. More complex internal energy functions, e.g. incorporating prior shape knowledge, have also been reported in literature [10,11].

The external energy is derived from the image, so that the snake will be attracted to features of interest. Given a grey level image $I(x, y)$, a common external energy is defined as:

$$
E_{\text {ext }}[\mathbf{r}]=\int-\mid \nabla I\left(\left.\mathbf{r}(s)\right|^{2} d s\right.
$$

or

$$
E_{\text {ext }}[\mathbf{r}]=\int-\left|\nabla\left(G_{\sigma}(x, y) * I(\mathbf{r}(s))\right)\right|^{2} d s
$$

where $\nabla$ is the gradient operator, $G_{\sigma}(x, y)$ a 2D Gaussian kernel with standard deviation $\sigma$ and where $*$ is the convolution operator.

\subsection{Optimization}

Eq. (1) can be minimized using gradient descent by treating $\mathbf{r}(s)$ as a function of time, i.e. $\mathbf{r}(s, t)$. The partial derivative of $\mathbf{r}$ with respect to $t$ is then

$$
\begin{aligned}
& \frac{d \mathbf{x}(s, t)}{d t}=\alpha \frac{d^{2} \mathbf{x}(s, t)}{d s^{2}}-\beta \frac{d^{4} \mathbf{x}(s, t)}{d s^{4}}-\frac{\partial E_{\text {ext }}}{\partial x} \\
& \frac{d \mathbf{y}(s, t)}{d t}=\alpha \frac{d^{2} \mathbf{y}(s, t)}{d s^{2}}-\beta \frac{d^{4} \mathbf{y}(s, t)}{d s^{4}}-\frac{\partial E_{e x t}}{\partial y}
\end{aligned}
$$

The snake stabilizes, i.e. an optimum is found, when the terms $\frac{d \mathbf{x}(s, t)}{d t}$ and $\frac{d \mathbf{y}(s, t)}{d t}$ vanish. 
This gradient descent approach requires a good initialization, close to the object boundary, in order to segment the object. This limitation is caused by the nature of the partial derivatives of the external energy, which differs from the null vector only in the proximity of the object's boundary. As we move away from the boundary these derivatives approach the null vector, or under the influence of noise point towards false optima. This results in a contour which will converge to a local optimum. To overcome this problem, $\mathrm{Xu}$ and Prince [12] proposed to replace the partial derivatives by an external force $\mathbf{v}(\mathbf{r}(s))=(u(\mathbf{r}(s)), v(\mathbf{r}(s)))$. This force is calculated by minimizing the following energy functional:

$$
\begin{aligned}
& E_{G V F}[u, v]= \\
& \qquad \int \mu\left(\frac{d u^{2}}{d x}+\frac{d u^{2}}{d y}+\frac{d v^{2}}{d x}+\frac{d v^{2}}{d y}\right)+|\nabla f|^{2}|\mathbf{v}-\nabla f|^{2} d x d y
\end{aligned}
$$

where $\mu$ is a nonnegative parameter expressing the degree of smoothness of the force field $\mathbf{v}$ and where $\mathrm{f}$ is an edge map, e.g. $f(x, y)=|\nabla I(x, y)|$. The first term of Eq. (5) keeps the field $\mathbf{v}$ smooth, whereas the second term forces the field $\mathbf{v}$ to resemble the original edge map in the neighbourhood of edges. This new external force is called gradient vector flow (GVF). For details on the optimization of Eq. (5), we refer to [12].

\section{Probabilistic active contours}

The active contour framework has already been proven useful for a wide range of applications. However, tuning the weighting parameters of the energy function remains a challenging task. The optimal parameters are a trade-off, where the regularization weights have to be set high enough to overcome the influence of clutter and low enough to accurately detect the true contour of the object. In this section, a new set of active contours is defined. This framework is based on statistical modelling of object features, thus omitting the tuning of the weighting parameters.

\subsection{Framework}

The goal of our active contour framework is to find the contour, $\mathbf{r}^{*}($.$) , which is most$ probable to delineate the object of interest. This can be formalized as finding the contour that maximizes $P[O[\mathbf{r}()]$.$] , where O[\mathbf{r}()$.$] is a predicate returning true if the contour$ delineates the object of interest and returns false if it does not. Let's assume that in order to find such a probable contour we can use a set of features $F(x, y)$ measured in the image, e.g. the edge strength at a specific pixel. The optimal contour can be defined as

$$
\mathbf{r}^{*}(.)=\underset{\mathbf{r}(.)}{\arg \max } P[O[\mathbf{r}(.)] \mid F(., .)]
$$


Using Bayes rule, we can rewrite this as

$$
\begin{aligned}
\mathbf{r}^{*}(.) & =\underset{\mathbf{r}(.)}{\arg \max } \frac{P[F(., .) \mid O[\mathbf{r}(.)]] P[O[\mathbf{r}(.)]]}{P[F(., .)]} \\
& =\underset{\mathbf{r}(.)}{\arg \max }\left(\log \frac{P(F(., .) \mid O[\mathbf{r}(.)])}{P(F(., .))}+\log P(O[\mathbf{r}(.)])\right)
\end{aligned}
$$

Equivalent to the snake energy, we can define an internal and external probability, respectively: $P_{\text {int }}[\mathbf{r}()]=.\log P(O[\mathbf{r}()]$.$) and P_{\text {ext }}[\mathbf{r}()]=.\log \frac{P(F(., .) \mid O[\mathbf{r}(.)])}{P(F(., .))}$.

\subsection{Internal probability}

The internal probability is completely independent of the image and can be used to incorporate the shape possibility of an object of interest. As an example we will use a simple model proposed in [13], where the likeliness of a contour only depends on the second derivative of the contour.

$$
\begin{aligned}
P_{\text {int }}[\mathbf{r}(.)] & =\log P[O[\mathbf{r}(.)]] \\
& =\log P\left(\left|\frac{\partial^{2} \mathbf{r}(s)}{\partial s^{2}}\right|\right] \\
& =\int_{0}^{1} \log P\left(\left|\frac{\partial \mathbf{r}^{2}(t)}{\partial t^{2}}\right|\right) d t
\end{aligned}
$$

For this last step we assume that the second derivative of $\mathbf{r}(\mathbf{t})$ is independent for every $t$. This off course assumes that the probability distribution of $P\left(\left|\frac{\partial \mathbf{r}(t)}{\partial t^{2}}\right|\right)$ is known. This probability distribution can be learned out of a small training set of ground truth segments. Note that this is just an example of an internal probability model. If the objects of interest has a specific shape or if they have more pronounced local features, e.g. jags, then the internal probability could be formulated using a more complex shape model, such as the models proposed in $[11,10]$.

\subsection{External probability}

The external probability depends on the image features that are used to characterize an object. As example we will model the objects of interest as an edge map, e.g. $F(x, y)=\mid$ $\nabla I(x, y) \mid$. If we consider the gradient to be independent for all $(x, y)$, then the external 
probability can be rewritten as:

$$
\begin{aligned}
P_{e x t}[\mathbf{r}(.)]= & \log \frac{P[F(., .) \mid O[\mathbf{r}(.)]]}{P[F(., .)]} \\
= & \log \frac{P[|\nabla I(., .)| \mid O[\mathbf{r}(.)]]}{P[|\nabla I(., .)|]} \\
= & \iint_{\Omega^{+}} \log \frac{P(|\nabla I(u, w)| \mid O[\mathbf{r}(.)])}{P(|\nabla I(u, w)|)} d u d w \\
& +\iint_{\Omega^{-}} \log \frac{P(|\nabla I(u, w)| \mid O[\mathbf{r}(.)])}{P(|\nabla I(u, w)|)} d u d w
\end{aligned}
$$

where $\Omega^{+}=\{(u, w) \mid(\exists k \in[0,1])(\mathbf{r}(k)=(u, w))\}$ and $\Omega^{-}=\{(u, w) \mid(\nexists k \in$ $[0,1])(\mathbf{r}(k)=(u, w))\}$. For the application of plant monitoring, imaging happens in a strictly controlled environment. Due to this controlled imaging, technicians can avoid clutter and thus minimize edges not coming from leaf contours.

Therefore $\iint_{\Omega^{-}} \log \frac{P(|\nabla I(u, w)| \mid O[\mathbf{r}(.)])}{P(|\nabla I(u, w)|)} d u d w$ will be very small. This allows us to approximate Eq. (11) by discarding this factor, i.e.

$$
P_{e x t}[\mathbf{r}(.)]=\int_{0}^{1} \log \frac{P(|\nabla I(\mathbf{r}(t))| \mid O[\mathbf{r}(.)])}{P(|\nabla I(\mathbf{r}(t))|)} d t
$$

The probabilities used in Eq. (12) can be interpreted as follows:

- $P(|\nabla I(\mathbf{r}(t))| \mid O[\mathbf{r}()]$.$) : the probability that the gradient of a point lying on the$ contour of a real segment is equal to $|\nabla I(\mathbf{r}(s))|$.

- $P(|\nabla I(\mathbf{r}(s))| \mid \mathbf{r}(s))$ : the probability that the gradient of a random point in the image is equal to $|\nabla I(\mathbf{r}(s))|$ whether or not this point is on the contour of a real object or not.

The probability distribution of the gradient strength of an object's contour can be estimated from a small training set of images where the objects are manually segmented. First measure the gradient strength at the contours delineating the ground truth segments. Then based on these measurements, calculate the probability distribution, e.g. using a kernel density estimator. The probability distribution of the gradient can be estimated in a similar way, but instead of measuring only the gradient strength at the contours, measure it at each pixel in the training data set. Note that although this example uses the gradient, this framework could also incorporate other image features such as ridges, intensity, output of a feature detector, region-based features, etc. $[1,14,6]$. 


\subsection{Optimization}

Substituting the proposed internal in external probabilities in Eq. (8) results in:

$$
\begin{aligned}
\mathbf{r}^{*}(.) & =\underset{\mathbf{r}(.)}{\arg \max }\left(P_{\text {int }}[\mathbf{r}(.)]+P_{\text {ext }}[\mathbf{r}(.))\right] \\
& =\underset{\mathbf{r}(.)}{\arg \max } \int_{0}^{1} \log \frac{P(|\nabla I(\mathbf{r}(s))| \mid O[\mathbf{r}(.)])}{P(|\nabla I(\mathbf{r}(s))|)}+\log P\left(\left|\frac{\partial \mathbf{r}^{2}(s)}{\partial s^{2}}\right|\right) d s
\end{aligned}
$$

This optimization can be solved using gradient descent by treating $\mathbf{r}(s)$ as a function of time, i.e. $\mathbf{r}(s, t)$. The partial derivative of $\mathbf{r}$ with respect to $t$ is then

$$
\begin{aligned}
& \frac{d x(s, t)}{d t}=\frac{d \log P\left(\left|\frac{\partial \mathbf{r}^{2}(s, t)}{\partial s^{2}}\right|\right)}{d s}+\frac{\partial \log \frac{P(|\nabla I(\mathbf{r}(s, t))| \mid O[\mathbf{r}(., t)]=\text { true })}{P(|\nabla I(\mathbf{r}(s, t))|)}}{\partial x} \\
& \frac{d y(s, t)}{d t}=\frac{d \log P\left(\left|\frac{\partial \mathbf{r}^{2}(s, t)}{\partial s^{2}}\right|\right)}{d s}+\frac{\partial \log \frac{P(|\nabla I(\mathbf{r}(s, t))| \mid O[\mathbf{r}(., t)]=\text { true })}{P(|\nabla I(\mathbf{r}(s, t))|)}}{\partial y}
\end{aligned}
$$

In order to use gradient descent, the initial $\mathbf{r}(s)$ should be in the vicinity of the true object. To avoid the probabilistic snake of converging to a local, false optimum, the same optimization technique as used with classical snakes can be used, i.e. optimization using gradient vector flow. This can easily be done by imposing the edge map in Eq. 5 to be $F(x, y)=\log \frac{P(|\nabla I(x, y)| \mid O(x, y)=\text { true })}{P(|\nabla I(x, y)|)}$, where $O(x, y)=$ true represents the assumption that $(x, y)$ lies on the contour of a leaf.

\section{Results}

The proposed method was developed to automate the measurement of average temperature of leaves. Therefore individual leaves should be segmented and tracked over time. The dataset used to validate the proposed method monitors sugar beet seedling plants using a thermal camera. The time-lapse sequences were captured at a time resolution of one image an hour. These thermal images are noisy, low contrast greyscale images. In these time-lapse sequences the 4 leaves of the sugar beet seedlings all move in different directions, at different speeds.

Fig. 1 shows an example of leaf segmentation using both the classical active contours as our proposed probabilistic active contours. All active contours are optimized using the gradient vector flow optimization. The gradient vector flow force was calculated using 30 iterations and a smoothing factor $\mu$ equal to 0.1 . In Fig.1 (a) the initialization of the four different snakes is shown. As can be seen, is this initialization already a good approximation of the real leaf contours. This proper initialization is however not sufficient for the classical active contours to converge to the real leaf boundaries. Two examples of active contour segmentation using different weighting parameters $\alpha$ and $\beta$ 
in Eq. (2) can be seen in Fig.1 (b) and (c). The active contours in Figure (b) were optimized using $\frac{1}{6}$ and $\frac{1}{3}$ respectively for weighting parameters $\alpha$ and $\beta$. These weighting values are apparently too low to prevent the contours to converge to false local optima. An example of such an incorrect convergence can be seen at the yellow contour, which partially converged towards the border of a wrong leaf. The cause of these segmentation errors is the difference in the gradient strength. Figure (d) shows the absolute value of the gradient in the image. The bigger leaves display a much stronger gradient which attract the contours of the elongated smaller leaves. Note this effect near the stalk of the "yellow" leaf. In Figure (c), the yellow contour converged correctly by increasing the contour weighting parameters, i.e. values $\frac{5}{3}$ and $\frac{2}{3}$ respectively for parameters $\alpha$ and $\beta$. However due to these strong smoothness constraints, the green and blue contours lose the real leaf borders near the tip of the leaves. Clearly, a general set of weighting parameter values is difficult to find. Even when such "optimal" parameter combination could be found for one image, it is unlikely that it would work for all the images in the sequence.

We now show results for our proposed method to illustrate that it does not suffer from the parameter selection, nor does it suffer from the linear influence that edge strength has. In order to use the proposed method, the internal and external probabilities have to be modelled first. The prior probabilities used for our method were learned using a single ground truth image. This image originated from a training time lapse sequence that was manually segmented. The distributions were calculated using the kernel density estimator with a normal distribution as kernel. In Fig.1 (e) the external probability at each pixel is shown. The gradient strength at leaf borders varies between leaves, nevertheless show the different leaves an equally strong probability at their border. This results in a better segmentation result as can be seen in Figure (f).

The previous example started from an almost perfect initialization. This was helpful to illustrate the problems that might occur with classical active contours, but it is rare that such a good initial contour is available. A more realistic example is shown in Fig. 2. The initialization is shown in Figure (a). In Figure (b) a detailed view of the GVF force field is shown. The force field points towards both leaves, as can be expected. The regularization effect of the internal probability however enforces the contour to converge to the correct leaf as is shown in Figure (c).

To quantitatively validate the proposed method, in 56 images have been manually segmented, each containing 4 leaves. The initialization of the active contours was based on these ground truth segments: the segments were dilated using a circular structuring element of size 5 , the borders of these dilated segments were then used as initialization. As a validation metric the Dice coefficient is used: consider $S$ the resulting segment from the active contour, i.e. the region enclosed by $\mathbf{r}(s)$, and GT the ground truth segment, then the Dice coefficient between $\mathrm{S}$ and GT is defined as:

$$
d(S, G T)=\frac{2 \operatorname{Area}(S \wedge G T)}{\operatorname{Area}(\mathrm{S})+\operatorname{Area}(G T)}
$$

Here $S \wedge G T$ consist of all pixels which both belong to the detected segment as well as to the ground truth segment. If $S$ and GT are equal, the Dice coefficient is equal to one. The Dice coefficient will approach zero if the regions hardly overlap. In order to compare our method with the active contours with the most optimal parameter setting, the image 


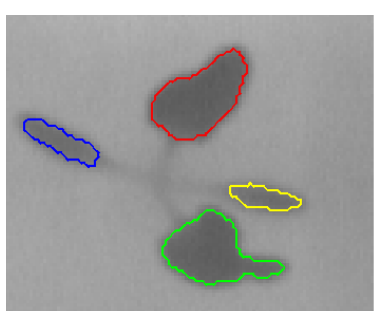

(a)

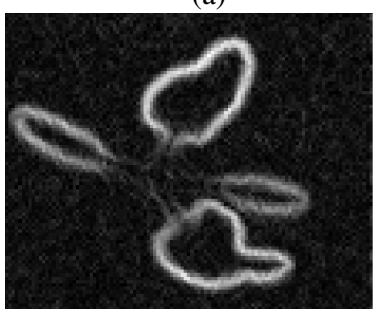

(d)

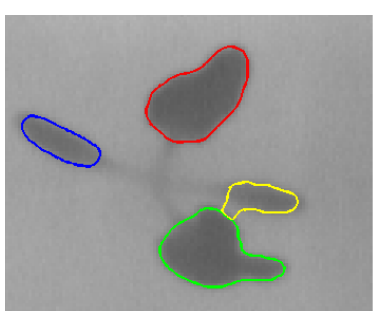

(b)

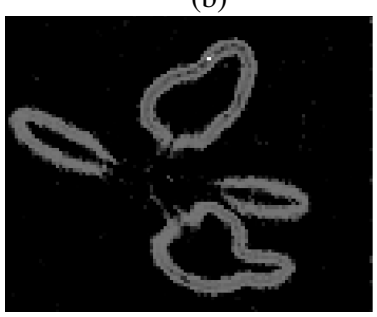

(e)

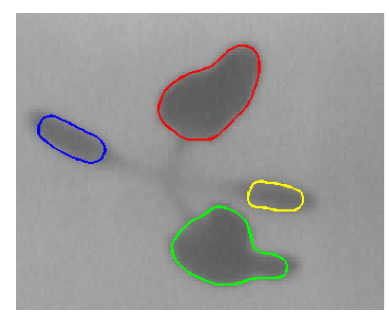

(c)

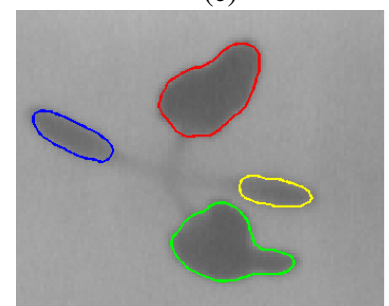

(f)

Fig. 1. Examples of segmentation using (probabilistic) active contours. The top row contains in (a) the initialization used for the (probabilistic) active contours, (b) the segmentation result of the classical active contours with $\alpha=\frac{1}{6}, \beta=\frac{1}{3}$, (c) the segmentation result of the classical active contours with $\alpha=1, \beta=\frac{2}{3}$. The bottom row: (d) the gradient strength of the image, (e) the external probability of each pixel in the image, (f) the segmentation result of the proposed probabilistic active contours

sequence has been segmented with $\alpha$ and $\beta$ both in the range of $0, \frac{1}{30}, \frac{2}{30}, \frac{3}{30}, \ldots, 1$. The best combination of $\alpha$ and $\beta$ resulted in an average Dice coefficient of 0.872 . Using these optimal parameters, 24 segments resulted in a Dice coefficient equal to 0 , i.e. the segments where completely lost. The proposed probabilistic active contours resulted in an average Dice coefficient of 0.929 with no leaves lost.

As a last example our proposed method was applied for leaf tracking in a time lapse sequence. Since the movement of the leaves does not seem to have a clear motion model, we cannot incorporate prior knowledge about the motion in our tracking methods such as in [15,1]. Instead the result of frame $t$ will be used as an initialization for frame $t+1$, such as done by Tsechpenakis et al. [16]. As can be seen in $n$ Fig. 3 does the proposed method cope with the movement and deformation of the leaves. Even frame 15 where the illumination level diminished due to nightfall, is still segmented correctly. If this illumination change is too strong, the learned probability distributions will not correspond to the image features. Therefore the segmentation results will be less accurate. This can already be seen at Figure (c), where the contours delineating the bigger leaves miss the true border at the center of the plant. Although this error is almost unnoticeable at this frame, there's a risk that it becomes more prominent in subsequent frames which will use these contours as an initialization. 


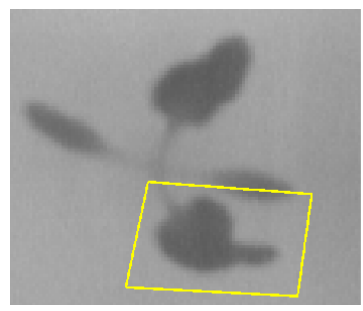

(a)

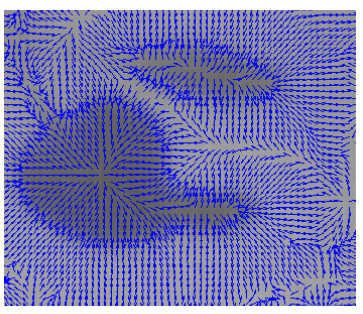

(b)

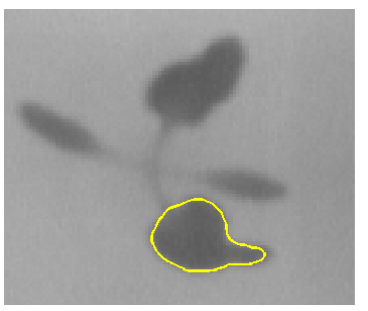

(c)

Fig. 2. Examples of segmentation using probabilistic active contours. (a) the initialization used for the (probabilistic) active contours, (b) the gradient vector flow of the external probability, i.e. the force used to optimize the external probability of the active contour (c) the segmentation result of the proposed probabilistic active contours

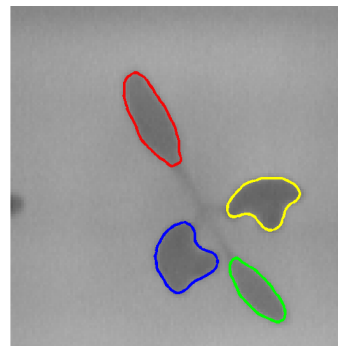

(a) frame 1

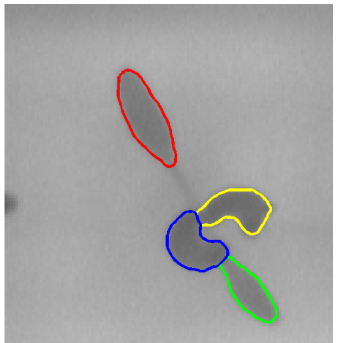

(b) Frame 7

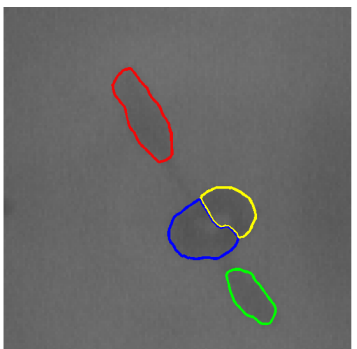

(c) Frame 15

Fig. 3. Example of tracking using probabilistic active contours 


\section{Discussion and Conclusion}

In this paper a new variant on the active contour framework is defined. Instead of optimizing an energy function it strives to maximize the probability that the contour is on the edge of an object. The proposed method does not need to tune a set of weighting parameters, since it is based on probability theory. This approach however needs a good estimate of the probability distribution functions that are needed for the calculation of the internal and external probability. These probability distributions can be learned from a ground truth training set. This method has been tested for the segmentation and tracking of sugar beet seedling leaves in thermal time lapse sequences. In these tests the proposed technique has been shown to be useful and outperformed classical active contours for the segmentation of multiple objects. To cope with changing light conditions, the learned probability distributions should be updated in order to follow the illumination settings of the image. This could be done using methods similar to background maintenance techniques [17]. We intend to investigate the influence of these methods in future research.

\section{Acknowledgment}

The authors are grateful to Erik De Bruyne, SESVanderhave NV, for providing the plant material. Jonas De Vylder is funded by the Institute for the Promotion of Innovation by Science and Technology in Flanders (IWT).

\section{References}

1. Isard, M., Blake, A.: Active contours. Springer (1998)

2. Liu, Y.: Automatic $3 \mathrm{~d}$ form shape matching using the graduated assignement algorithm. pattern recognition 38 (2005) 1615-1631

3. Kass, M., Witkin, A., Terzopoulos, D.: Snakes: active contour models. International journal of computer vision (1988) 321-331

4. Tsechpenakis, G., Rapantzikos, K., Tsapatsoulis, N., Kollias, S.: A snake model for object tracking in natural sequences. Signal Processing-Image Communication 19(3) (2004) 219 238

5. Charmi, M.A., Derrode, S., Ghorbel, F.: Fourier-based geometric shape prior for snakes. Pattern Recognition Letters 29(7) (2008) 897-904

6. Chan, T., Vese, L.: An active contour model without edges. Scale-Space Theories in Computer Vision 1682 (1999) 141-151

7. Goldenberg, R., Kimmel, R., Rivlin, E., Rudzsky, M.: Fast geodesic active contours. Ieee Transactions on Image Processing 10(10) (2001) 1467-1475

8. Chan, T.F., Esedoglu, S., Nikolova, M.: Algorithms for finding global minimizers of image segmentation and denoising models. Siam Journal on Applied Mathematics 66(5) (2006) 1632-1648

9. Bresson, X., Esedoglu, S., Vandergheynst, P., Thiran, J.P., Osher, S.: Fast global minimization of the active contour/snake model. Journal of Mathematical Imaging and Vision 28(2) (2007) 151-167

10. Charmi, M.A., Derrode, S., Ghorbel, S.: Fourier-based geometric shape prior for snakes. Pattern Recognition Letters 29 (2008) 897-904 
11. Staib, L., Duncan, J.: Boundary finding with parametrcally deformable models. IEEE Transactions on Pattern Analysis and Machine Intelligence 14 (1992) 1061-1075

12. Xu, C., Prince, J.: Snakes, shapes and gradient vector flow. IEEE transactions on image processing 7 (1998) 359-369

13. Xu, C., Yezzi, A., Prince, J.: On the Relationship between Parametric and Geometric Active Contours. Proc. of 34th Asilomar Conference on Signals, Systems, and Computers 34 (October 2000) 483-489

14. Poon, C.S., Braun, M.: Image segmentation by a deformable contour model incorporating region analysis. Physics in Medicine and Biology 42 (sep 1997) 1833-1841

15. Goobic, A., Welser, M., Acton, S., Ley, K.: Biomedical application of target tracking in clutter. Proc. 35th Asilomar Conference on Signals, Systems and Computers 1 (2001) 88-92

16. Tsechpenakis, G., Rapantizikos, K., Tsapatsoulis, N., Kollias, S.: A snake model for object tracking in natural sequences. Signal processing: image communication 19 (2004) 219-238

17. Shireen, Khaled, Sumaya: Moving Object Detection in Spatial Domain using Background Removal Techniques - State-of-Art. Recent Patents on Computer Science 1 (2008) 32-34 